Objectives To compare the clinical outcomes of CABG and PCI with drug-eluting stent (DES) among patients with 2-vessel or 3-vessel coronary artery disease and CKD.

Methods Between January 2005 and June 2006, patients undergoing CABG or PCI with DES for treatment of 2 -vessel or 3-vessel coronary disease were evaluated for renal function by eGFR calculated using modified MDRD equation. Patients undergoing incomplete revascularisation with $\mathrm{PCI}$ were excluded. CKD was defined as eGFR $<60 \mathrm{ml} / \mathrm{min}$. all the participants were followed up for 2 yrs. The primary end point of follow-up was the composite of all-cause death, non-fatal myocardial infarction (MI), or cerebrovascular events (CVE). The second end point was repeat revascularisation.

Results After the exclusion of patients undergoing incomplete revascularisation with PCI, 409 patients received complete revascularisation for treatment with 2-vessel disease and 415 with 3 -vessel disease were evaluated. In the 2 -vessel population, the incidence of primary end point and second end point were both very similar in patients receiving DES and CABG (primary end point: $9.3 \%$ vs $8.2 \%, p=0.753$; second end point: $6.1 \%$ vs $3.1 \%, p=0.253$ ). In the 3 -vessel population, there was also no significant difference in the frequency of primary end point $(11.4 \%$ vs $10.5 \%, p=0.774)$ between DES $(n=167)$ and CABG groups $(n=248)$. However, patients receiving DES still experienced significantly higher rate of repeat revascularisation as compared with patients who underwent CABG during 2-year follow-up $(10.2 \%$ vs $4.4 \%, p=0.022)$. Multivariate Cox regression analysis of long-term outcome showed that the choice of revascularisation strategy were not an independent predictor of repeat revascularization in patients with 2-vessel disease (HR $1.52,95 \%$ CI 0.53 to $3.98, p=0.387$ ). However, PCI with DES was independently associated with higher risk of repeat revascularisation in patients with 3 -vessel disease compared with CABG (HR 2.32, 95\% CI 1.57 to $7.33, \mathrm{p}=0.024$ ).

Conclusion Compared with CABG, PCI with drug-eluting stent showed similar incidence of death, MI or cerebrovascular events in patients with multivessel disease and CKD, but is associated with increased repeat revascularisation in 3-vessel population, even after complete revascularisation.

\section{e0504 CHRONIC KIDNEY DISEASE AND THE RISK OF STENT THROMBOSIS AFTER PERCUTANEOUS CORONARY INTERVENTION WITH DRUG-ELUTING STENTS}

doi:10.1136/hrt.2010.208967.504

Wang Zhijian, Zhou Yujie, Zhao Yingxin, Liu Yuyang, Shi Dongmei, Gao Fei. Anzhen Hospital Capital Medical University

Background Chronic kidney disease (CKD) has been demonstrated to be associated with adverse clinical outcomes for patients with coronary heart disease. However, data on relation of CKD and stent thrombosis after drug-eluting stent (DES) implantation is limited. Objectives This study was designed to examine whether CKD is associated with higher incidence of stent thrombosis after elective coronary drug-eluting stent (DES) implantation compared with patients with normal renal function.

Methods We consecutively enrolled 2972 patients undergoing elective percutaneous coronary intervention (PCI) with DES. Demographic and clinical data were collected preoperatively. CKD was defined as estimated glomerular filtration rate (eGFR) $<60 \mathrm{ml} / \mathrm{min}$, calculated using the modified $\mathrm{MDRD}$ equation. The primary outcome was 1-year definite or probable stent thrombosis.

Results Five hundred and ninety four participants (19\%) had CKD before procedure. The incidence of 1-year definite or probable stent thrombosis was significantly higher in CKD patients compared with patients with normal renal function $(1.7 \%$ vs $0.5 \%, p=0.001)$. After adjustment for multiple clinical and biochemical covariates, CKD was an independent predictor of 1-year definite or probable stent thrombosis (HR 3.26, 95\% CI 1.74 to $8.37, \mathrm{p}=0.009$ ).
Conclusion CKD is significantly associated with increased incidence of 1-year definite or probable stent thrombosis in patients undergoing PCI with DES.

\section{e0505 COMPARISON OF THE CLINICAL APPLICATION BETWEEN HOME-MADE FIREBIRD2TM DRUG-ELUTING STENT AND IMPORTED ENDEAVOR-SPRINT DRUG-ELUTING STENT}

doi:10.1136/hrt.2010.208967.505

\section{Li Ping. Guangxi Yulin first People's Hospital}

Background The research and development of drug-eluting stents are mainly concentrates in countries of Europe and America presently, but it is encouraging that the home-made drug-eluting stents are invented and recommended constantly in recent years. For example, Firebird sirolimus drug-eluting stent has been got a widespread application in china today, and its safety and effectiveness are generally acknowledged. Firebird2 sirolimus drug-eluting Co-based alloy supporting system is the second generation DES which is researched and developmented independently by ShangHai MicroPort medical instrument Corporation. It had got the registered certificate in January 15, 2008. It is a new generation drug-eluting stent that bases on the cobalt-based alloy platform, completely new stent structures, a better biological compatibility, and two-layer SBS polymer coating sirolimus drug-eluting stent, which have higher safty and effectiveness. This study was made in the base of Clinical application registration, making a comparison with that of the imported ENDEAVOR-Sprint drug-eluting stent, and makes a further observation on its safety and effectiveness.

Objectives To make a comparison on the safty and effectiveness of home-made Firebird2 sirolimus drug-eluting Co-based alloy stent with that of the imported ENDEAVOR-Sprint drug-eluting stent.

Methods 100 Coronary artery disease cases that Accept interventional therapy were divided into Firebird group (50 cases) and Endeavor group (50 cases) Randomly, the curative effect was compared between two groups.

Results The average ages, sex ratio, hypertension, diabetes mellitus, and myocardial infarction history in two groups have no statistical differentiation. One-vessel, two-vessel and three vessel lesion, bifurcation lesions, chronic total occlusion, and left main lesions in two groups have no statistical differentiation ( $p>0.05)$. The average Vascular diameter and target Lesion length in two groups have no statistical differentiation $(p>0.05)$. The operation success rate were $100 \%$ in both groups. No thrombosis event happened in Firebird group, one subacute thrombosis event happened in Endeavor group, which a revascularisation was needed. 28 cases in Firebird group were accepted reexaminnation of the coronary angiography (CAG) and one got restenosis, and 2 got restenosis in 19 cases which accepted reexaminnation of CAG in Endeavor group, there was no statistical differentiation between two groups ( $p>0.05)$.

Conclusions We can obtain a favourable safty and satisfying near and medium-term curative effects from the implantation of homemade Firebird2 sirolimus drug-eluting Co-based alloy stents in CHD cases, and it has no statistical differentiation when compared with that of the imported ENDEAVOR-Sprint drug-eluting stent.

\section{C0506 APPLICATION OF OVERLAPPED SIROLIMUS-ELUTING STENTS IN TREATMENT OF LONG CORONARY ARTERIES LESION}

doi:10.1136/hrt.2010.208967.506

Mu Lei, Liu Huiliang, Ma Dongxing, Luo Jianping, Han Wei, Yang Yong. Department of Cardiologygeneral Hospital of Capf

Objective To evaluate the clinical efficacy of overlapped Excel and Cypher stents in treatment for long coronary arteries lesion. 
Method 374 patients with de-novo long coronary arteries lesion who performed 2 Excel or Cypher stents which diameter varied $2.5 \mathrm{~mm} \sim 3.5 \mathrm{~mm}$ in department of cardiology, general hospital of CAPF were enrolled in from 2006.8 to 2009.3 retrospectively. These patients were divided to two groups by the type of stents implanted: Excel group and Cypher group. The incidence of composite MACE and stent thrombosis during hospitalisation and 12-month clinical follow-up outside hospital after PCI between two groups were compared, follow-up angiograpy were performed in several patients, and in-stent LLL, in-segemt LLL and incidence of restenosis between two groups were compared.

Result Clinical characteristics and characteristics of target lesion were similar between two groups, PCI procedure were similar except the ratio of pre-dilatation between two groups. Incidence of composite MACE during hospitalisation after PCI of Excel group and Cypher group were $5.7 \%$ and $6.1 \%$, incidence of composite MACE 12-month clinical follow-up outside hospital were $6.6 \%$ and $8.8 \%$ respectively, incidence of acute/subacute stent thrombosis defined by ARC of Excel group and Cypher group were $2.8 \%$ and $2.5 \%$, incidence of late stent thrombosis were $2.7 \%$ and $2.7 \%$ respectively, there were no significant differences between two groups; incidence of in-stent restenosis of Excel group and Cypher group follow-up were $6.0 \%$ and $9.6 \%$, incidence of in-segment restenosis were $9.5 \%$ and $12 \%$ respectively, that revealed nonsignificant differences between two groups; in-segment LLL of Excel group and Cypher group were $0.11 \pm 0.06 \mathrm{~mm}$ and $0.12 \pm 0.08 \mathrm{~mm}$, there were no significant differences between two groups, but instent LLL were $0.09 \pm 0.05 \mathrm{~mm}$ and $0.10 \pm 0.05 \mathrm{~mm}$ respectively, that showed significant difference between two groups $(\mathrm{p}=0.037)$.

Conclusion The safety and efficacy of overlapped Excel stents were similar as overlapped Cypher stents in treatment of long coronary arteries lesion, meanwhile, overlapped Excel stents relieve in-stent LLL than overlapped Cypher stents; LLL of overlapped site was more severe than non-overlapped site, whatever Excel or Cypher stents, but there was no significant difference in ratio of restenosis between overlapped site and any other regions.

\section{e0507 COMPOSITION OF THE SAFETY AND FEASIBILITY BETWEEN 4F AND 5F CATHETER IN TRANSRADIAL CORONARY ANGIOGRAPHY}

doi:10.1136/hrt.2010.208967.507

Liang Jing, Zhou Yujie, Liu Xiaoli. Department of Cardiology, Beijing Anzhen Hospital, Capital Medical University, Beïing, China

Objectives To explore the efficacy and feasibility of the transradial approach for diagnostic coronary angiography with $4 \mathrm{~F}$ and $5 \mathrm{~F}$ catheter.

Methods All patients undergoing a coronary angiogram the first time were eligible to be included in the study. They were randomised to either 4F or $5 \mathrm{~F}$ catheters. The success rate of angiography, image quality and vascular complications between two groups were compared. Two-dimensional and colour Doppler ultrasonic examination was performed on the right radial artery before procedures, 1 day and 4 weeks after procedures, respectively.

Results A total of 847 consecutive patients were randomised to $4 \mathrm{~F}$ catheter group ( $\mathrm{n}=446)$, including $266(63.4 \%)$ men and 5F universal catheter group ( $\mathrm{n}=401)$, including $271(67.6 \%)$ men. There were no significant difference between $4 \mathrm{~F}$ catheter and $5 \mathrm{~F}$ universal catheter group in success rate of angiography (98.6\% vs 97.1\%), image quality $(2.83 \pm 2.1$ vs $2.88 \pm 2.31$ ( $p>0.05)$ ). Both groups had no death, acute thrombosis and major bleeding. The radial artery spasm and radial artery occlusion was more often on women. Intimamedia thickness of radial artery had significantly increased among female patients after procedures.
Conclusion With low complication rate and better tolerance, 4F catheter should be selected in the diagnostic coronary angiography by transradial approach, especially for small built female patients.

\section{e0508 COMPARATIVE ANALYSIS OF DIFFERENT STRATEGY FOR REVASCULARIZATION ON EFFENCY IN OLD WOMAN DIABETIC PATIENTS WITH COMPLEX CORONARY DISEASE}

doi:10.1136/hrt.2010.208967.508

Ge Hailong, Zhou Yujie, Zhao Yinxin, Shi Dongmei, Liu Yuyang, Guo Yonghe, Yang Qing, Cheng Wanjun. Anzhen Hospital, Capital Medical University, Beijing, China

Objectives To evaluate and analysis the clinical efficacy and prognosis of two different strategy of revascularization by percutaneous coronary intervention (PCI) with drug-eluting stent (DES) and coronary artery bypass graft (CABG) in old woman diabetic patients with complex coronary disease.

Background Elder, Female and Diabetic is markeble risk factor for poor prognosis after PCI and CABG. Which strategy of revascularization (PCI vs $\mathrm{CABG}$ ) and the influence factor that could promote the choice of strategy for revascularization may be result in better outcome is uncertain in these patients. Further evaluations in adequately data are awaited to confirm the clinical benefit of two strategy.

Methods 523 female patients whose age were above 75 years old with DM, multivessel disease underwent PCI (206) or CABG (317) were included studied. The choice of revascularization was dependent on clinical baseline and procedural characteristics of patients and/or physician recommendation. Major adverse cardiac events (MACE) included death, myocardial infarction and repeat coronary revascularization.

Results MACE rates at 1 year is equivalent between $\mathrm{CABG}$ and $\mathrm{PCI}$ (2.1\% vs $4.2 \%$ (OR $1.8 ; \mathrm{p}>0.5$ ). There was a similar risk of the combined endpoints of death $(1.5 \%$ vs $2.8 \%)$, myocardial infarction $(1.2 \%$ vs $1.6 \%)$ and cerebrovascular events (1.1\% vs $2.3 \%)$ at 1 year, but the risk of target vessel revascularisation (TVR) was significantly higher $(1.8 \%$ vs $7.8 \%, \mathrm{p}<0.01)$. Compared with PCI patients, the lesions seem more complex. the prevalence of calcified lesions, total occlusion, collateral circulation were higher in CABG patients $(p<0.01)$. The number of the diseased vessels was the only independent predictors of type of revascularization (PCI vs CABG).

Conclusions PCI with DES placement was safe compared to CABG as a type of revascularization in elder female patients with DM and MVD at 1-year. A clinical strategy for revascularization by PCI or CABG should be carefully assessed.

\section{e0509 EFFENCY OF TRIPTOLIDE-COATED STENT ON PREVENT RESTENOSIS}

doi:10.1136/hrt.2010.208967.509

Ge Hailong, Zhou Yujie, Ma Hanying, Liu Xiaoli, Wang Zhijian, Yang Shiwei, Nie Bin, Jia Dean. Department of Cardiology, Anzhen Hospital, Capital Medical University, China

Objective The aim of this study is to test the efficacy and safety of triptolide-coated stent on anti-inflammatory and inhibiting intimal hyperplasia compared with that of a drug eluting stent and a bare metal stent in a pig after angioplasty.

Methods 15 triptolide-coated stents (TCS), 12 drug eluting stent (Cypher select) and 12 bare metal stents (BMS) were deployed with oversizing (stent/artery ratio 1.1:1) in a porcine coronary arteries. Coronary angiography, histopathologic and inflammatory markers levels were carried out and analysed at week 4 after stenting. 